Supporting Information

\title{
Bioinspired Superwettable Microspine Chips with Directional Droplet Transportation for Biosensing
}

Yanxia Chen, ${ }^{\dagger}, \#$ Kan Li, ${ }^{\ddagger}, \#$ Shudong Zhang, ${ }^{\perp}, \#$ Lei Qin, ${ }^{\dagger}$ Shaohui Deng, ${ }^{\perp}$ Liyuan Ge ${ }^{\perp}$ Li-Ping Xu, ${ }^{*}, \S$

Lulin Ma, ${ }^{*}{ }^{\perp}$ Shutao Wang, ${ }^{*}$, and Xueji Zhang*, $\|$

${ }^{\S}$ Research Center for Bioengineering and Sensing Technology, University of Science and Technology Beijing, Beijing 100083, China

${ }^{\dagger}$ Beijing Key Laboratory for Sensor, Beijing Key Laboratory for Optoelectronic Measurement Technology, Key Laboratory of Modern Measurement \& Control Technology, Ministry of Education, Beijing Information Science and Technology University, Beijing, 100101, China

${ }^{\ddagger}$ CAS Key Laboratory of Bio-inspired Materials and Interfacial Science, CAS Center for Excellence in Nanoscience, Technical Institute of Physics and Chemistry, Chinese Academy of Sciences, Beijing 100190, China

${ }^{\perp}$ Department of Urology, Peking University Third Hospital, Beijing 100191, China

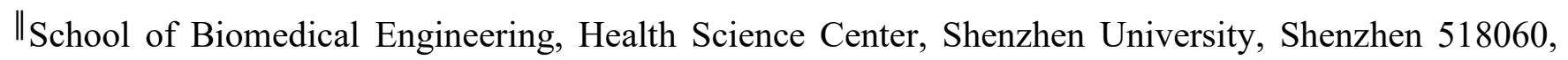
Guangdong, China

E-mail: $\quad$ xuliping@ustb.edu.cn, malulin@medmail.com.cn, stwang@mail.ipc.ac.cn, zhangxueji@szu.edu.cn 


\section{Materials}

Tetraethyl orthosilicate (98\%, TEOS, Alfa Aesar), n-octadecyltrichlorosilane (95\%, OTS, Acros Organics), (3-mercaptopropyl) trimethoxysilane (95\%, Alfa Aesar), N-ymaleimidobutyryloxy succinimide ester ( $\geq 98 \%$, GMBS, Sigma-Aldrich), methylene blue trihydrate (95\%, Acros Organics). Phosphate-buffered saline (PBS, $10 \mathrm{mM}, \mathrm{pH}$ 7.4). Human prostate-specific antigen (PSA), two mouse

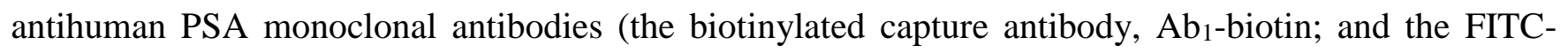
labeled PSA antibody, Ab2-FITC) were purchased from Shanghai Linc-Bio Science Co. LTD. Streptavidin (SA) was purchased from Amresco Commercial Finance, LLC. Bovine serum albumin (BSA) was from Sigma Co. Milli-Q water (Millipore Corp, 18.2 M $\mathrm{cm}$ ) was used for all aqueous solutions.

\section{Instruments and Characterization}

A field-emission scanning electron microscope (JSM-6700F, Japan) was used for characterizing the morphologies of the nanodendritic coatings. Water contact angles (WCAs) and behaviors of droplet transportation were captured by an OCA-20 system (Data-Physics, Germany) at ambient temperature. The fluorescence images were captured by inverted fluorescence microscopy (Nikon, Ti-E). The optical images were obtained by digital camera 60D (Canon Co., Ltd). 
a)

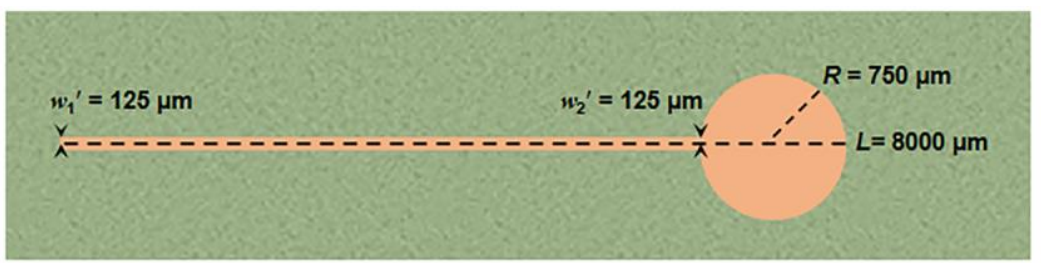

b)

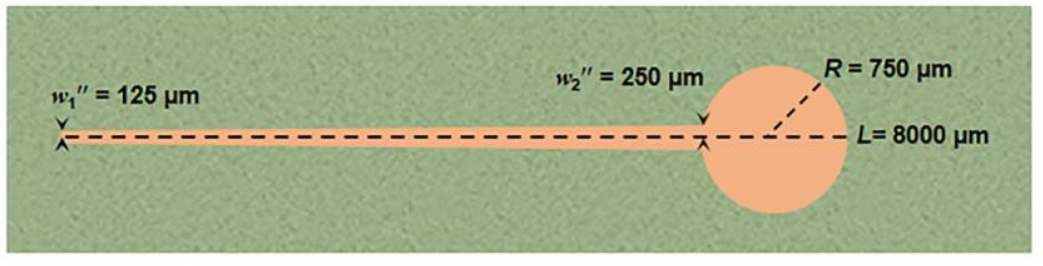

c)

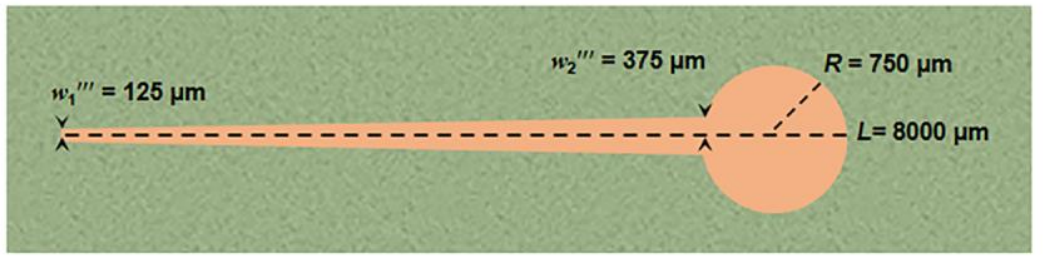

Figure S1. Three SMS chips with different geometric asymmetry. The lengths $(L)$ of these SMS chips are $8000 \mu \mathrm{m}$, and the radii $(R)$ of microwells are $750 \mu \mathrm{m}$. The half-widths of microchannels are the same at the narrow end $\left(w_{1}{ }^{\prime}=w_{1}{ }^{\prime \prime}=w_{1}{ }^{\prime \prime \prime}=125 \mu \mathrm{m}\right)$ and different at the broad end $\left(w_{2}{ }^{\prime}=125 \mu \mathrm{m}, w_{2}{ }^{\prime \prime}=250 \mu \mathrm{m}\right.$, $\left.w_{2}^{\prime \prime \prime}=375 \mu \mathrm{m}\right)$, and the corresponding semiapex angles ( $\alpha$ of eq 3 ) of these gradient microchannels are $0^{\circ}, 1.1^{\circ}$, and $2.2^{\circ}$, respectively.

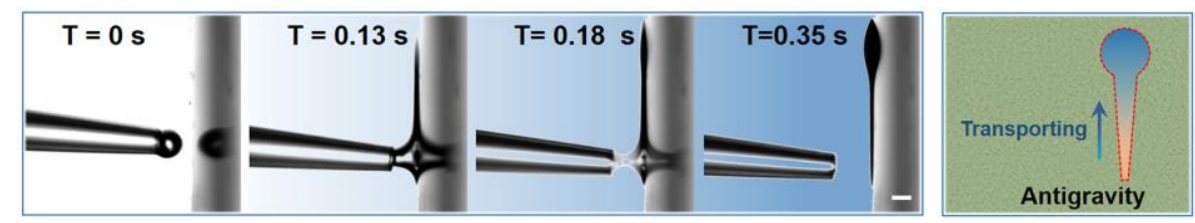

Figure S2. Spontaneous and directional water droplet transportation on the SMS chip even antigravity. Scar bar: $1 \mathrm{~mm}$.
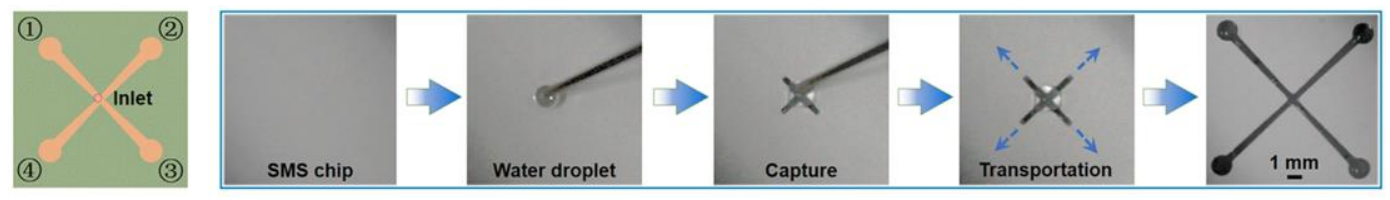

Figure S3. Directional water droplet transportation on the multimicrochannel SMS chip. 


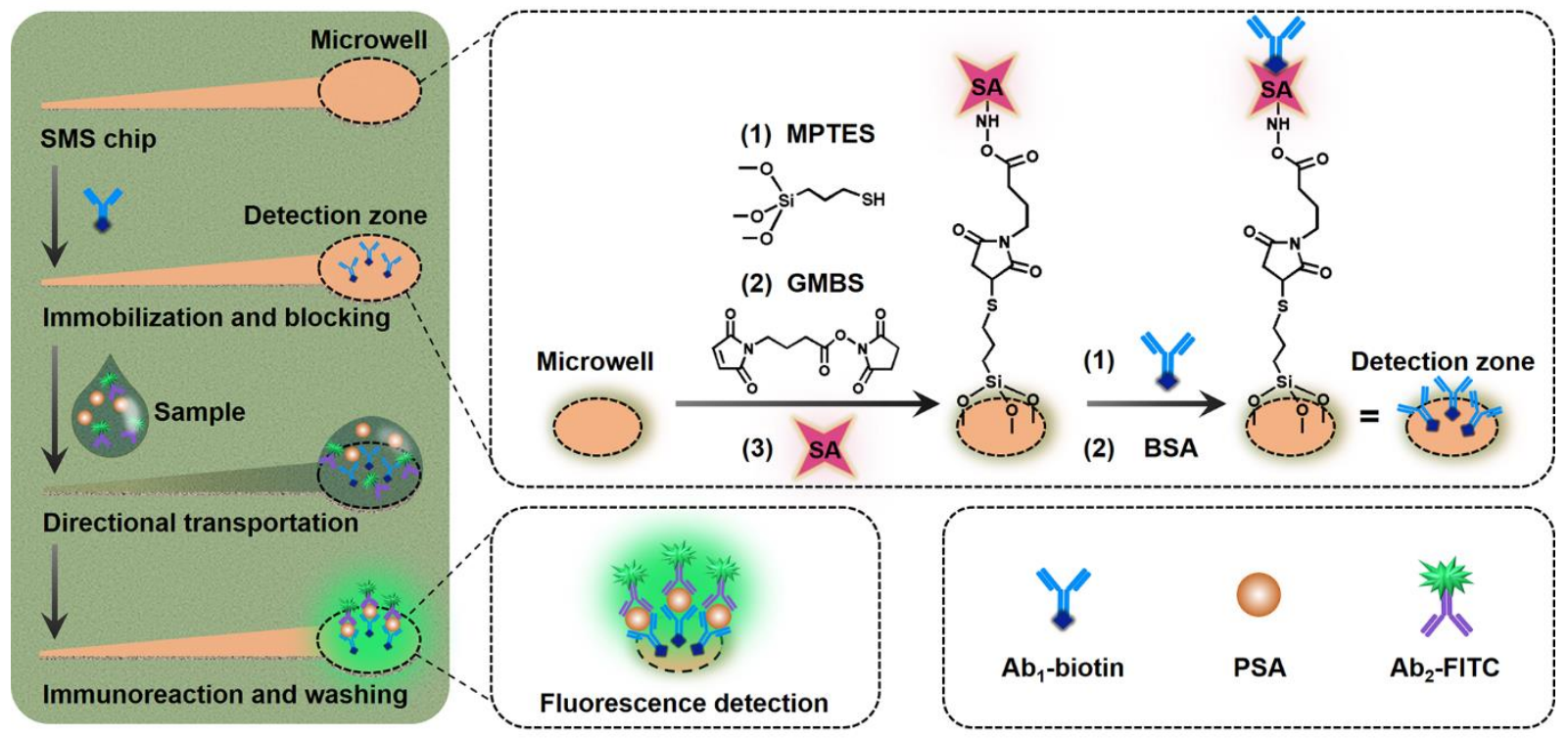

Figure S4. Schematic illustration of the fluorescence detection of PSA on the SMS chip.

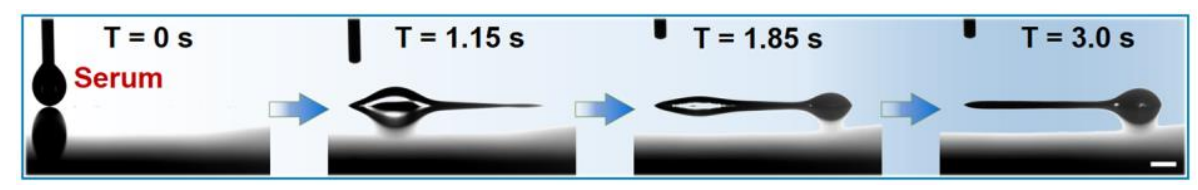

Figure S5. Directional transportation of serum $(2 \mu \mathrm{L})$ on the SMS chip. Scar bar: $1 \mathrm{~mm}$. 